\title{
Increased asymmetric dimethylarginine and nitric oxide levels in patients with migraine
}

\author{
Ertugrul Uzar • Osman Evliyaoglu • Gülten Toprak • \\ Abdullah Acar • Yavuz Yucel • Tugba Calisir • \\ Mehmet Ugur Cevik $\cdot$ Nebahat Tasdemir
}

Received: 4 October 2010/Accepted: 14 November 2010/Published online: 27 February 2011

(C) The Author(s) 2011. This article is published with open access at Springerlink.com

\begin{abstract}
Asymmetric dimethylarginine (ADMA) has been found as correlated with endothelial dysfunction and oxidative stress. There are few studies regarding ADMA and nitric oxide (NO) levels in patients with migraine and alterations of ADMA and NO levels during migraine attack are not well-known. Therefore, in present study, we aimed to measure NO and ADMA levels in patients with migraine and compare them with the control group to investigate the correlation between migraine, oxidative stress and endothelial dysfunction. The migraine group consisted of 59 patients, including 22 suffering from migraine with aura and 37 suffering from migraine without aura. The control group consisted of 31 healthy volunteers without headache. The patients in migraine group were divided into subgroups based on whether attack period was present or not and whether it was migraine with or without aura. Plasma ADMA levels were measured using an enzyme-linked immunosorbent assay method. Migraine patients had higher concentrations of $\mathrm{NO}(35.6 \pm 7.7,31.0 \pm 6.2 \mu \mathrm{mol} / \mathrm{L}$, respectively, $p=0.005)$ and ADMA (0.409 \pm 0.028 , $0.381 \pm 0.044 \mu \mathrm{mol} / \mathrm{L}$, respectively, $p=0.001)$ levels when compared with the healthy controls. During migraine attack, NO and ADMA levels were found to be significantly higher in migraine group as compared to control group (respectively, $p=0.015, p=0.014$ ). Similarly, NO and
\end{abstract}

E. Uzar $(\bowtie) \cdot$ A. Acar · Y. Yucel · T. Calisir .

M. U. Cevik · N. Tasdemir

Department of Neurology, Faculty of Medicine,

Dicle University School of Medicine, Dicle University,

21280 Diyarbakir, Turkey

e-mail: ertuzar@yahoo.com

O. Evliyaoglu · G. Toprak

Department of Biochemistry, Faculty of Medicine,

Dicle University, Diyarbakir, Turkey
ADMA levels in the patients with migraine in the interictal period were found to be significantly higher as compared to control group $(p=0.011, p=0.003)$. In conclusion, higher ADMA and NO levels of patients with migraine supported that oxidative stress and endothelial dysfunction may have a role in migraine pathogenesis.

Keywords Migraine - Asymmetric dimethylarginine · Nitric oxide - Oxidative stress - Endothelial dysfunction

\section{Introduction}

Migraine is a common, chronic and disabling neurovascular disorder that characterized by severe headache attacks with photophobia, nausea, vomiting, autonomic symptoms, and in some patients with aura involving neurological symptoms. Although the pathogenesis of migraine is still unclear, different vascular, neurological, and neuroinflammatory mechanisms have been suggested [1, 2]. It has been reported that nitric oxide (NO) plays a role during trigeminovascular inflammation occurring during migraine attacks [2]. Nitric oxide, which is formed by the constitutive isoforms of $\mathrm{NO}$ synthase, endothelial nitric oxide synthase (eNOS) and neuronal nitric oxide synthase (nNOS), plays an important roles in the regulation of cerebral blood flow and cell viability and in the protection of nerve cells or fibers against pathogenic factors associated with neurological disorders [3]. Enhanced endothelial NO and superoxide anion release may cause migraine through cerebral blood flow changes. In some studies, oxidative stress due to increased NO has been found especially during attacks in patients with migraine [4, 5]. It is emphasized that neurogenic inflammation, endothelial dysfunction and oxidative stress have a role in migraine pathogenesis [1-5]. 
Nitric oxide is one of the indicators of oxidative stress [5]. Asymmetric dimethylarginine (ADMA) is nitric oxide synthase inhibitor and ADMA has close association with endothelial dysfunction and oxidative stress [6]. There is limited number of studies investigating ADMA levels in patients with migraine $[7,8]$. No difference has been found in ADMA and NO levels between migraine patients during interictal period and healthy controls in a previous study [7]. However, ADMA levels had not been investigated in patients with migraine attack. The association between ADMA and NO in migraine attack is not well-known [7]. For these purposes, in present study, the relationships between migraine, oxidative stress and endothelial dysfunction were investigated by comparing NO and ADMA levels in migraine patients with headache attack and interictal period.

\section{Patients and methods}

A total of 59 (40 women and 19 men) consecutive newly diagnosed migraine patients who did not receive any prophylactic migraine medication were included in this study. The study group consisted of 22 patients, suffering from migraine with aura and 37 migraine patients without aura that followed-up in the outpatient clinic, Department of Neurology, Dicle University Hospital. Migraine was diagnosed based on the criteria of the International Headache Classification II [9]. The patients in migraine group were divided into subgroups as migraine in the attack period (with and without aura) $(n=24)$ and migraine in the interictal period (with and without aura) $(n=35)$. Age range of the patients was 18-57 years (mean \pm standard deviation: $33.3 \pm 9.4$ years). Patients who had hypertension (known hypertension treated with antihypertensive drugs, two or more blood pressure recordings greater than 140/90 mm Hg), coronary artery disease (angiographically proven coronary lesions $>50 \%$ or documented myocardial infarction, angina pectoris, previous percutaneous coronary intervention or coronary artery bypass grafting), diabetes mellitus (known diabetes treated with diet or drugs or both; or either a fasting serum glucose of more than $126 \mathrm{mg} / \mathrm{dl}$ ), stroke and renal disease were excluded from the study. The control group included 31 healthy participants without a history of headache, vascular diseases, hypertension, diabetes mellitus, and renal and cardiac diseases. The control group comprises 19 women and 12 men. Mean age of the control group was $30.7 \pm 8$ years (range, $18-50$ years). Informed consent was obtained from all participants and the study protocol was approved by the local ethics committee. Blood sampling was performed within $2-5 \mathrm{~h}$ from the onset of migraine headache or headache-free period in the patients with migraine patients. Similarly, blood sampling was also performed in healthy individuals. Each collected blood sample was immediately centrifuged at $4,000 \mathrm{rpm}+4{ }^{\circ} \mathrm{C}$ for $10 \mathrm{~min}$ and then transferred into an eppendorf tube. Samples were transferred on ice and kept at $-80^{\circ} \mathrm{C}$ deep freeze until the end of the study which was completed in 3 months. The method for plasma nitrite and nitrate levels was based on the Griess reaction [10]. As NO measurement is very difficult in biological specimens, because it is readily oxidized to nitrite $\left(\mathrm{NO}^{2-}\right)$ and subsequently to nitrate $\left(\mathrm{NO}^{3-}\right)$ which serves as index parameters of NO production. Griess method was used as the principle of the NO assay after the protein was removed from the samples by salt precipitation, and the $\mathrm{NO}^{3-}$ in the sample was reduced to $\mathrm{NO}^{2-}$ by activated cadmium granules, which was equal to the NO concentration of the sample [10]. The plasma ADMA levels were determined using the enzyme-linked immunosorbent assay (Immundiagnostik; Bensheim, Germany) method as previously described [11].

Statistical analysis

All statistical analyses were performed using the Statistical Package for the Social Sciences (SPSS), version 11.5, for Windows (SPSS, Chicago, IL, USA). Data were expressed as mean \pm standard deviation. The normality of the distribution for all variables was assessed by the Kolmogorov-Smirnov test. Student's $t$ test was used for normally distributed variables and Mann-Whitney $U$ test was used for non-parametric variables. Relationships between variables were analyzed by Pearson or Spearman correlation analysis according to distribution type of parameters. A $p<0.05$ was considered to be statistically significant.

\section{Results}

Some demographical and biochemical variables of the study and control groups are shown in Table 1. No differences were found in mean age, body mass index (BMI), blood pressure and levels of fasting blood glucose, cholesterol, triglyceride, LDL and HDL between two groups $(p>0.05)$. The NO and ADMA concentrations of the study subjects are shown in Table 2. Migraine patients had higher concentrations of $\mathrm{NO}(35.6 \pm 7.7$ vs. $31.0 \pm 6.2 \mu \mathrm{mol} / \mathrm{L}$, respectively, $p=0.005)$ and ADMA $(0.409 \pm 0.028,0.381 \pm$ $0.044 \mu \mathrm{mol} / \mathrm{L}$, respectively, $p=0.001)$ when compared with healthy controls. NO and ADMA concentrations of the migraine subgroups are shown in Table 3. No statistically significant difference was found between NO (35.3 \pm 6.4 , $35.8 \pm 8.5 \mu \mathrm{mol} / \mathrm{L}$, respectively, $p>0.05)$ and ADMA $(0.407 \pm 0.025, \quad 0.410 \pm 0.031 \mu \mathrm{mol} / \mathrm{L}, \quad$ respectively, $p>0.05)$ levels during attack and interictal period in 
Table 1 Demographical and biochemical variables of the study and control groups

N.S. statistically not significant $(p>0.05), B M I$ body mass index

\begin{tabular}{llll}
\hline & Migraine patients $(n=59)$ & Controls $(n=31)$ & $p$ values \\
\hline Age (years) & $33.1 \pm 9.9$ & $30.7 \pm 8.1$ & N.S. \\
Sex $(\mathrm{M} / \mathrm{F})$ & $19 / 40$ & $12 / 19$ & N.S. \\
HDL-C $(\mathrm{mg} / \mathrm{dl})$ & $48.4 \pm 13.6$ & $50.1 \pm 8.8$ & N.S. \\
LDL-C $(\mathrm{mg} / \mathrm{dl})$ & $102.1 \pm 23.8$ & $95.7 \pm 34.1$ & N.S. \\
Cholesterol $(\mathrm{mg} / \mathrm{dl})$ & $174.6 \pm 32.4$ & $176.8 \pm 31.2$ & N.S. \\
Fasting glucose (mg/dl) & $92.7 \pm 6.1$ & $91.2 \pm 4.9$ & N.S. \\
Systolic blood pressure $(\mathrm{mmHg})$ & $108.3 \pm 12.3$ & $106.1 \pm 10.8$ & N.S. \\
BMI $\left(\mathrm{kg} / \mathrm{m}^{2}\right)$ & $26.2 \pm 2.6$ & $27.1 \pm 3.2$ & N.S. \\
\hline
\end{tabular}

migraine. NO is involved in the regulation of the cerebral vessels tone. NO may function as a signaling molecule in controlling neuronal activity and is important in controlling sensory inputs during migraine attack and interacting with reactive oxygen substances (ROS), which may induce headache through changes of cerebral blood flow [12]. The most important effect of NO is the activation of the soluble guanylate cyclase. This enzyme causes the synthesis of cyclic guanosine monophosphate (cGMP) and NO-cGMP pathway in smooth vascular muscles causes vascular dilation and relaxation $[13,14]$. It is suggested that NO could be an important mediator in the initiation or the propagation of a neurogenic cranial vessel inflammatory response that might eventually result in a migraine attack [14]. It has been found that the pathways consisting of NO synthesis are activated in the experimental headache models such as nitroglycerin-induced headache [15]. The increase of NO may be caused by interactions with free ROS. The primary product of the interaction between $\mathrm{NO}$ and superoxide anion is peroxynitrite. Peroxynitrite is an aggressive and potent cellular oxidant [16]. So, increased NO with migraine patients may be associated with oxidative stress. In addition, occurrence of peroxynitrite following NO increase affects endothelia function [17]. Marked NO increase in migraine group in our study strengthened the correlation between oxidative stress and endothelia dysfunction in pathophysiology of migraine. Likewise, Yllmaz et al. [5] suggested that NO may serve as useful markers to show the increased oxidative stress in migraine patients. Taffi et al. [18] found significant increased peroxynitrite as a potent oxidant in platelets of patients with migraine without aura during headache-free periods. It has been reported that the migraine headache and the changes in
Table 3 Comparison of nitric oxide (NO) and asymmetric dimethylarginine (ADMA) levels of migraine subgroups

N.S. statistically not significant $(p>0.05)$

\begin{tabular}{llll}
\hline & Migraine during attack $(n=25)$ & Interictal period $(n=24)$ & $p$ \\
\hline NO $(\mu \mathrm{mol} / \mathrm{L})$ & $35.3 \pm 6.4$ & $35.8 \pm 8.5$ & N.S. \\
ADMA $(\mu \mathrm{mol} / \mathrm{L})$ & $0.407 \pm 0.025$ & $0.410 \pm 0.031$ & N.S. \\
& Migraine with aura $(n=22)$ & Without aura $(n=37)$ & P \\
NO $(\mu \mathrm{mol} / \mathrm{L})$ & $35.0 \pm 6.5$ & $35.9 \pm 8.4$ & N.S. \\
ADMA $(\mu \mathrm{mol} / \mathrm{L})$ & $0.406 \pm 0.017$ & $0.410 \pm 0.033$ & N.S. \\
\hline
\end{tabular}


cerebral blood flow during migraine attacks may be related to the production of NO $[16,19]$. Also, Shimomura et al. [20] found that high concentrations of platelet nitrate/nitrite ratio during migraine attacks. But, Shukla et al. [21] found no changes in polymorphonuclear leukocyte, platelet and plasma nitrite levels in patients with migraine. Additionally, it is suggested that intranasal hydroxocobalamin, a NO scavenger, may have a prophylactic effect in migraine [22]. Highly increased NO levels of our migraine patients during attack and interictal period as compared to the controls are in concordance with the results of the most studies [16-20].

In recent studies, a relationship between migraine, stroke and cardiovascular diseases have been reported [23, 24]. Endothelial dysfunction is induced by numerous cardiovascular risk factors and represents an early event in the development of atherosclerosis [25]. ADMA are elevated in several disease states, including those characterized by endothelial dysfunction such as stroke, cardiovascular diseases, hypertension, pre-eclampsia, atherosclerosis, hypercholesterolemia, diabetes mellitus, and chronic kidney disease $[25,26]$. It is suggested that endothelial dysfunction has a role in the pathogenesis of correlation between migraine and stroke [24]. Therefore, in our study, ADMA level, a marker of endothelial dysfunction, was measured in migraine cases. To the contrary of two previously conducted studies, ADMA levels were found to be significantly higher in migraine cases compared to the control group in our study $[7,8]$. This finding supports the roles of oxidative stress and endothelial dysfunction in migraine pathogenesis. Our study is the first study determining ADMA elevations in migraine. Possible reasons for increase in levels of both $\mathrm{NO}$ and ADMA in the patients with migraine in our study may be as follows: (1) decrease in activity of dimethylarginine dimethylaminohydrolase (DDAH) enzyme, metabolizing ADMA and/or (2) excess amounts of ADMA could have been produced to compensate the abnormally increased NO in the body. It has been demonstrated that DDAH activity is regulated by NO [8, 27]. This implies that under certain conditions when NO generation increases, DDAH activity may be diminished. This would lead to accumulation of ADMA and inhibition of NO synthase [26]. Therefore, it is conceivable that an increase in ADMA in migraine might represent a compensatory mechanism to decrease NO production and consequently, to counterbalance excessive vasodilatation. It is suggested that endothelial injury, impaired endothelial vasoreactivity and increased carotid artery intima media thickness occur with migraine and these are associated with vascular risk factors [28]. In some studies, particularly migraine with aura has been found to be associated with endothelial dysfunction. It has been reported that there is evidence of increased endothelial activation in premenopausal women with migraine, particularly in those with migraine with aura, [29]. In a previous study, an eNOS (Glu298Asp) gene polymorphism decreasing eNOS activity has been determined more frequently in migraine patients with aura [30]. In our study, ADMA levels of migraine patients both with and without aura were found to be markedly higher than those of the healthy controls. When ADMA levels of migraine patients with and without aura were compared, slightly higher but not significantly different ADMA levels were found in patients without aura. These findings indicate the possible endothelial dysfunction in migraine patients both with and without aura. Findings of increased ADMA and NO in migraine may lead to new therapeutic strategies including probable antioxidant use for management of migraine In the future, development and enhancements of existing drugs may be accompanied by increased efforts to develop truly new migraine drugs based on knowledge of the pathophysiology [31, 32].

In conclusion, increased ADMA and NO levels of migraine patients support the roles of oxidative stress and endothelial dysfunction in migraine pathogenesis. Further clinical and biochemical studies are needed to investigate the associations between migraine, stroke and ADMA and to introduce new therapeutic modalities for migraine treatment.

\section{Conflict of interest None.}

Open Access This article is distributed under the terms of the Creative Commons Attribution License which permits any use, distribution and reproduction in any medium, provided the original author(s) and source are credited.

\section{References}

1. Silva FA, Rueda-Clausen CF, Silva SY, Zarruk JG, Guzmán JC, Morillo CA, Vesga B, Pradilla G, Flórez M, López-Jaramillo P (2007) Endothelial function in patients with migraine during the interictal period. Headache 47:45-51

2. Sarchielli P, Alberti A, Vaianella L, Pierguidi L, Floridi A, Mazzotta G, Floridi A, Gallai V (2004) Chemokine levels in the jugular venous blood of migraine without aura patients during attacks. Headache 44:961-968

3. Toda N, Ayajiki K, Okamura T (2009) Cerebral blood flow regulation by nitric oxide in neurological disorders. Can J Physiol Pharmacol 87:581-594

4. Ciancarelli I, Tozzi-Ciancarelli MG, Di Massimo C, Marini C, Carolei A (2003) Urinary nitric oxide metabolites and lipid peroxidation by-products in migraine. Cephalalgia 23:39-42

5. Yilmaz G, Sürer H, Inan LE, Coskun O, Yücel D (2007) Increased nitrosative and oxidative stress in platelets of migraine patients. Tohoku J Exp Med 211:23-30

6. Tain YL, Kao YH, Hsieh CS, Chen CC, Sheen JM, Lin IC, Huang LT (2010) Melatonin blocks oxidative stress-induced increased asymmetric dimethylarginine. Free Radic Biol Med (in press)

7. Guldiken B, Demir M, Guldiken S, Turgut N, Ozkan H, Kabayel L, Tugrul A (2009) Asymmetric dimethylarginine and nitric oxide levels in migraine during the interictal period. J Clin Neurosci 16:672-674 
8. Gruber HJ, Bernecker C, Lechner A, Weiss S, Wallner-Blazek M, Meinitzer A, Höbarth G, Renner W, Fauler G, Horejsi R, Fazekas F, Truschnig-Wilders M (2009) Increased nitric oxide stress is associated with migraine. Cephalalgia 30:486-492

9. Lima MM, Padula NA, Santos LC, Oliveira LD, Agapejev S, Padovani C (2005) Critical analysis of the international classification of headache disorders diagnostic criteria (ICHD I-1988) and (ICHD II-2004), for migraine in children and adolescents. Cephalalgia 25:1042-1047

10. Cortas NK, Wakid NW (1990) Determination of inorganic nitrate in serum and urine by a kinetic cadmium-reduction method. Clin Chem 36:1440-1443

11. Schulze F, Wesemann R, Schwedhelm E, Sydow K, Albsmeier J, Cooke JP, Böger RH (2004) Determination of asymmetric dimethylarginine (ADMA) using a novel ELISA assay. Clin Chem Lab Med 42:1377-1383

12. Ciancarelli I, Tozzi-Ciancarelli MG, Di Massimo C, Olivieri L, Carolei A (2005) Preventive non-pharmacological treatment and nitric oxide in chronic migraine. J Headache Pain 6:341-342

13. Olesen $\mathbf{J}$ (2008) The role of nitric oxide (NO) in migraine, tension-type headache and cluster headache. Pharmacol Ther 120:157-171

14. Napoli R, Guardasole V, Zarra E, Matarazzo M, D'Anna C, Saccà F, Affuso F, Cittadini A, Carrieri PB, Saccà L (2009) Vascular smooth muscle cell dysfunction in patients with migraine. Neurology 72:2111-2114

15. Sarchielli P, Alberti A, Codini M, Floridi A, Gallai V (2000) Nitric oxide metabolites, prostaglandins and trigeminal vasoactive peptides in internal jugular vein blood during spontaneous migraine attacks. Cephalalgia 20:907-918

16. Gruber HJ, Bernecker C, Pailer S, Fauler G, Horejsi R, Möller R, Lechner A, Fazekas F, Truschnig-Wilders M (2009) Hyperinsulinaemia in migraineurs is associated with nitric oxide stress. Cephalalgia 30:593-598

17. Förstermann U (2008) Oxidative stress in vascular disease: causes, defense mechanisms and potential therapies. Nat Clin Pract Cardiovasc Med. 5:338-349

18. Taffi R, Vignini A, Lanciotti C, Luconi R, Nanetti L, Mazzanti L, Provinciali L, Silvestrini M, Bartolini M (2005) Platelet membrane fluidity and peroxynitrite levels in migraine patients during headache-free periods. Cephalalgia 25:353-358

19. Fidan I, Yüksel S, Ymir T, Irkeç C, Aksakal FN (2006) The importance of cytokines, chemokines and nitric oxide in pathophysiology of migraine. J Neuroimmunol 171:184-188
20. Shimomura T, Murakami F, Kotani K, Ikawa S, Kono S (1999) Platelet nitric oxide metabolites in migraine. Cephalalgia 19:218-222

21. Shukla R, Barthwal MK, Srivastava N, Nag D, Seth PK, Srimal RC, Dikshit M (2001) Blood nitrite levels in patients with migraine during headache-free period. Headache. 41:475-481

22. Van der Kuy PH, Merkus FW, Lohman JJ, ter Berg JW, Hooymans PM (2002) Hydroxocobalamin, a nitric oxide scavenger, in the prophylaxis of migraine: an open, pilot study. Cephalalgia 22:513-519

23. Bigal ME, Kurth T, Santanello N, Buse D, Golden W, Robbins M, Lipton RB (2010) Migraine and cardiovascular disease: a population-based study. Neurology 74:628-635

24. Kurth $\mathrm{T}$ (2010) The association of migraine with ischemic stroke. Curr Neurol Neurosci Rep 10:133-139

25. Siekmeier R, Grammer T, März W (2008) Roles of oxidants, nitric oxide, and asymmetric dimethylarginine in endothelial function. J Cardiovasc Pharmacol Ther 13:279-297

26. De Gennaro Colonna V, Bianchi M, Pascale V, Ferrario P, Morelli F, Pascale W, Tomasoni L, Turiel M (2009) Asymmetric dimethylarginine (ADMA): an endogenous inhibitor of nitric oxide synthase and a novel cardiovascular risk molecule. Med Sci Monit 15:91-101

27. Dayoub H, Rodionov RN, Lynch C, Cooke JP, Arning E, Bottiglieri T, Lentz SR, Faraci FM (2008) Overexpression of dimethylarginine dimethylaminohydrolase inhibits asymmetric dimethylarginine-induced endothelial dysfunction in the cerebral circulation. Stroke 39:180-184

28. Hamed SA, Hamed EA, Ezz Eldin AM, Mahmoud NM (2010) Vascular risk factors, endothelial function, and carotid thickness in patients with migraine: relationship to atherosclerosis. J Stroke Cerebrovasc Dis 19:92-103

29. Tietjen GE, Herial NA, White L, Utley C, Kosmyna JM, Khuder SA (2009) Migraine and biomarkers of endothelial activation in young women. Stroke 40:2977-2982

30. Borroni B, Rao R, Liberini P, Venturelli E, Cossandi M, Archetti S, Caimi L, Padovani A (2006) Endothelial nitric oxide synthase (Glu298Asp) polymorphism is an independent risk factor for migraine with aura. Headache 46:1575-1579

31. Stovner LJ, Tronvik E, Hagen K (2009) New drugs for migraine. J Headache Pain 10:395-406

32. Farinelli I, De Filippis S, Coloprisco G, Missori S, Martelletti P (2009) Future drugs for migraine. Intern Emerg Med 4:367-373 